\title{
Cardiovascular risk reduction following metabolic and bariatric surgery
}

\author{
Wayne J. English, Matthew D. Spann, Chetan V. Aher, D. Brandon Williams \\ Department of Surgery, Vanderbilt University Medical Center, Nashville, TN, USA \\ Contributions: (I) Conception and design: All authors; (II) Administrative support: All authors; (III) Provision of study materials or patients: All \\ authors; (IV) Collection and assembly of data: All authors; (V) Data analysis and interpretation: All authors; (VI) Manuscript writing: All authors; (VII) \\ Final approval of manuscript: All authors. \\ Correspondence to: Wayne J. English. Department of Surgery, Vanderbilt University Medical Center, Nashville, TN, USA. Email: wayne.english@vumc.org.
}

\begin{abstract}
Cardiovascular disease (CVD) is the world's leading cause of mortality and obesity is a wellrecognized risk factor of CVD. Early detection and management of CVD is critical to reduce CVD risk. Especially in patients suffering from obesity with obesity-related CVD risk factors such as hypertension (HTN), dyslipidemia, and diabetes mellitus (DM). A substantial and sustained decrease in body weight after metabolic and bariatric surgery is associated with a significant reduction of cardiovascular risk factors. This article reviews CVD risk models, mechanisms of CVD risk associated with obesity, and overall CVD risk reduction between different metabolic and bariatric procedures.
\end{abstract}

Keywords: Cardiovascular risks; metabolic and bariatric surgery; risk reduction; cardiovascular risk reduction

Submitted Nov 25, 2019. Accepted for publication Jan 14, 2020.

doi: $10.21037 /$ atm.2020.01.88

View this article at: http://dx.doi.org/10.21037/atm.2020.01.88

\section{Introduction}

Cardiovascular disease (CVD) is the world's leading cause of mortality, representing approximately $31 \%$ of all deaths, or approximately 18 million people annually. Obesity has been well established as a major risk factor of CVD. The World Health Organization (WHO) emphasizes the need for early detection and management of CVD in patients suffering from obesity and other medical risk factors such as hypertension (HTN), dyslipidemia, and diabetes mellitus (DM). Addressing behavioral risk factors such as tobacco use, unhealthy diet, physical inactivity, and harmful use of alcohol can also result in profound improvements in CVD (1).

Modest weight loss in patients suffering from obesity produces improvement in type 2 diabetes mellitus (T2D), HTN and dyslipidemia [hypertriglyceridemia, insufficient high-density lipoprotein (HDL) cholesterol, and excessive low-density lipoprotein (LDL)] cholesterol; however, nonsurgical weight loss trials have failed to demonstrate any benefit in terms of CVD events in individuals suffering from obesity (2-5).
Alternatively, metabolic and bariatric procedures demonstrate significant CVD risk reduction and mortality reduction, while often completely eliminating numerous comorbidities and improving quality of life (6). The aim of this publication is to review CVD risk models, CVD risk associated with obesity, and overall CVD risk reduction between different metabolic and bariatric procedures.

\section{Risk factors for CVD and models used for calculating CVD risk}

Population-based CVD risk models focusing on traditional risk factors have been developed into guidelines aimed to improve cardiovascular (CV) health. Table 1 shows the different risk assessment tools being used worldwide, with risk factors measured to determine CVD risk (7-13).

The Framingham study identified age (males $\geq 45$ years or females $\geq 55$ years), gender (male sex), HTN, dyslipidemia, smoking, and DM as risk factors for developing coronary heart disease (CHD). The initial Framingham Risk Score (FRS) was developed in 1998 as a means to assess CHD 
Table 1 Risk prediction models used to calculate CVD risk

\begin{tabular}{|c|c|c|}
\hline Risk model & Risk assessment & Risk factors \\
\hline Framingham Risk Score & 10-year risk of $\mathrm{CHD}$ & $\begin{array}{l}\text { Age, sex, LDL-C, HDL-C, SBP, DBP, DM status, smoking status, } \\
\text { hypertension treatment }\end{array}$ \\
\hline QRISK $^{\circledR}$ 3-2018 Calculator & 10-year risk of $\mathrm{Ml}$ or stroke & $\begin{array}{l}\text { Age, sex, ethnicity, C/HDL-C ratio, smoking status, angina in } 1^{\text {st }} \\
\text { degree relative, CKD (stage } 3 / 4 / 5) \text {, hypertension treatment, RA, } \\
\text { SLE, mental illness, steroid treatment, erectile dysfunction, BMI }\end{array}$ \\
\hline $\begin{array}{l}\text { United Kingdom Prospective } \\
\text { Diabetes Study (UKPDS) }\end{array}$ & $\begin{array}{l}10 \text {-year risk of nonfatal, and fatal, } \\
\text { stroke and } \mathrm{CHD}\end{array}$ & $\begin{array}{l}\text { Age, sex, ethnicity, SBP, DM status, smoking status, TC, HDL, A1C, } \\
\text { atrial fibrillation }\end{array}$ \\
\hline $\begin{array}{l}\text { Registre Gironí del Cor } \\
\text { (REGICOR) }\end{array}$ & 10-year risk of Ml, mortality & Age, sex, SBP, DBP, TC, HDL-C, DM status, smoking status \\
\hline \multicolumn{3}{|c|}{$\begin{array}{l}\text { ACC/AHA/ASCVD, American College of Cardiology/American Heart Association/Atherosclerotic Cardiovascular Disease; CV, } \\
\text { cardiovascular; TC, total cholesterol; HDL-C, high density lipoprotein-cholesterol; LDL-C, low density lipoprotein cholesterol; SBP, systolic } \\
\text { blood pressure; DBP, diastolic blood pressure; DM, diabetes mellitus; C, cholesterol; CKD, chronic kidney disease; RA, rheumatoid } \\
\text { arthritis; SLE, systemic lupus erythematosus; BMI, body mass index; TG, triglyceride; MI, myocardial infarction; hsCRP, high sensitivity } \\
\text { C-reactive protein; CHD, coronary heart disease; CVD, cardiovascular disease. }\end{array}$} \\
\hline
\end{tabular}

risk and was refined by the ATP-III in 2002 with a focus on CHD endpoints, mortality, and nonfatal myocardial infarction. The 2008 Framingham General CVD Risk Score incorporated additional CV endpoints including stroke, heart failure, and peripheral arterial disease (14-16).

The 2013 ACC/AHA (American College of Cardiology/ American Heart Association) Pooled Cohort Atherosclerosis Cardiovascular Disease (ASCVD) Risk Score is now widely used and is derived from several studies: the Framingham original and offspring cohorts, the Atherosclerosis Risk in Communities (ARIC) study, the Coronary Artery Risk Development in Young Adults (CARDIA) study, and the Cardiovascular Health Study (CHS) (17-20).

Other multivariate risk models have been developed and incorporate different variables including $\mathrm{HbA1C}$ levels, atrial fibrillation, chronic kidney disease, erectile dysfunction in males, and systemic inflammation such as rheumatoid arthritis or systemic lupus. Family history of premature atherosclerotic CVD (age $<55$ years for males and $<65$ years for females) has also been included in some of the risk assessment models. Of note, limitations exist with all CVD risk models, as some individuals who develop CVD may have few, if any, known risk factors (21). Also challenging is the fact that more than one-third of individuals with HTN in the United States are undiagnosed, and only $30-40 \%$ of patients with elevated low-density lipoprotein cholesterol (LDL-C) are prescribed lipidlowering therapies $(14,21)$. Understanding these limitations can aid in patient counselling.

\section{Concepts of CVD risk reduction}

There are modifiable and non-modifiable risk factors associated with CVD. Modifiable risk factors include a sedentary lifestyle, tobacco use, unhealthful diet, obesity, HTN, T2D, and dyslipidemia. Traditionally, health care providers have focused on lifestyle interventions and medications to reduce CVD risk. Recently however, a concept has emerged of using surgical alterations of the gastrointestinal tract to reduce CVD risk. This category of 
surgery is referred to as metabolic surgery because of the myriad of metabolic benefits that have been demonstrated to occur. This type of surgery is also known as bariatric surgery because the primary goal has historically been weight loss. The current era of surgery for weight loss has proven to be a safe, effective and durable for patients with class 2 and 3 obesity. However, support for current procedures may be inappropriately withheld due to bias against historical procedures often linked to significant morbidity and mortality. For example, patients undergoing jejunoileal bypass were subjected to developing significant nutritional complications and cirrhosis. Additionally, undesirable morbidity and mortality was seen prior to and early in the laparoscopic era of bariatric surgery. Bariatric surgery has since evolved from being a procedure primarily designed for weight loss to now being the best available treatment option for improving the metabolic burden of obesity. Most obesity-related comorbidities significantly improve or completely resolve after surgery, particularly factors associated with CVD risk, such as diabetes and insulin resistance, hemodynamic and inflammatory parameters, dyslipidemia, and adiposity with its associated pathophysiology (22).

One of the most common directives that patients are given to reduce CVD risk is to increase physical activity. Studies show that performing more than 150 minutes of moderate physical activity, or 75 minutes of vigorous physical activity, every week can achieve a 30\% CVD risk reduction, and walking at least two hours a week reduced the incidence of premature death from CVD by about $50 \%$ $(23,24)$. However, patients with severe obesity often suffer from physical limitations due to severe joint and back pain, which can ultimately lead to a sedentary lifestyle; thus, generating a vicious cycle of inactivity, low exercise capacity, further weight gain, and ultimately, increased mortality risk. By halting and then reversing the vicious cycle of obesity and impaired exercise tolerance, weight loss after bariatric surgery leads to increased physical activity levels. Reduction of body weight after LRYGB surgery is associated with significantly improved cardiorespiratory function 6 months after surgery, especially in patients who lost more than $18 \%$ of their initial body weight (25). A meta-analysis of 26 studies looked at changes after bariatric surgery in self-reported physical activity and objectively measured cardiovascular endurance (26). All but one study reported an increase in self-reported physical activity 12 months after surgery. Patients were physically active for a greater period of time, but at a lower intensity in the first 6 months following surgery; this was demonstrated by a reduction in moderate to vigorous activity but an increase in daily step count on average of 1,225-2,749 more steps per day. All studies assessing endurance testing, either by treadmill or timed walking tests, reported improvements after bariatric surgery.

Another highly frequent goal in the effort to reduce CVD risk is weight loss. In particular, waist circumference, especially when due to excess visceral fat, is more strongly correlated to the development of metabolic syndrome and, ultimately, adverse CV outcomes than body mass index (BMI) (27-29). Excess visceral adiposity has been linked to many CVD risk factors including dyslipidemia, insulin resistance, hyperinsulinemia, glucose intolerance, altered fibrinolysis and endothelial dysfunction. Even at a healthy BMI, excess visceral adiposity is associated with a harmful increased accumulation of ectopic fat in the heart, liver, and skeletal muscle, which might contribute to increased atherosclerosis and CVD risk (30). Visceral fat has a strong effect on CVD risk, partially since it impairs immune function and alters leukocyte counts and cell-mediated immune responses (31). Visceral adipocyte enlargement also triggers endoplasmic reticulum stress and hypoxia, which stimulates the expression of inflammatory genes and activates immune cells. In addition, increased leptin (proinflammatory mediator) and the reduction in adiponectin (anti-inflammatory mediator) activate immune cells. Bariatric surgery has been shown to reduce visceral fat with subsequent improvement of leukocytosis, inflammation and endothelial dysfunction. A systematic review demonstrated a significant reduction in $\mathrm{C}$-reactive protein (CRP), interleukin-6, tumor necrosis factor- $\alpha$ (TNF- $\alpha$ ) levels after bariatric surgery (32). LRYGB has been shown to markedly improve inflammatory markers (interleukin-6 and C-reactive protein) and endothelial function (Intracellular adhesion molecule-1, tissue-type plasminogen activator antigen and von Willebrand factor). These improvements were sustained 24 months post-surgery (33). LRYGB and laparoscopic sleeve gastrectomy (LVSG) significantly lowered high sensitivity-CRP levels for patients with elevated CVD risk (34). Erythrocyte sedimentation rate, which is a known predictor of coronary heart disease, is also improved post-surgery $(35,36)$.

\section{CVD risk improvement after bariatric surgery}

Currently, LVSG is the most common metabolic and bariatric surgery procedure performed, $46 \%$ of all 
procedures, in the world. In the United States, LVSG represents $60 \%$ of all bariatric procedures $(37,38)$. However, in a 2011 systematic review of 52 studies looking at CVD risk, most of the patients underwent open and LRYGB $(45.8 \%)$ with only $0.2 \%$ undergoing LVSG. The review demonstrated a $40 \%$ relative risk reduction of CVD and striking remission rates, or improvement, for HTN, T2D and dyslipidemia (68\%, 75\% and $71 \%$ respectively) (39). Current data analyzing CVD risk and bariatric surgery since this review have demonstrated similar results in single institution studies. While there is a lack of CVDspecific randomized control trials, several randomized controlled studies looking primarily at patients receiving medical versus surgical treatment for T2D demonstrated a significant improvement in CVD risk $(40,41)$.

An observational study by Adams and colleagues looked at HTN, T2D and dyslipidemia in LRYGB patients with 12-year follow-up, compared to nonsurgical patients who sought to have surgery but did not primarily for insurance reasons, and a third group of nonsurgical patients who did seek to undergo surgery. This study showed longterm durability of weight loss and effective remission and prevention of T2D, HTN, and dyslipidemia after LRYGB. Each one of the CVD risk factors was significantly better in the surgery group compared to the non-surgery control groups. In particular, T2D, low levels of HDL-C, and high triglyceride levels were each nearly eliminated from the LRYGB group (42).

Another randomized study further demonstrated the superiority of surgical over medical therapy in CVD risk reduction in obese patients with T2D. Three years after randomization between bariatric surgery and intensive medical weight management, surgical patients had greater weight loss, lower HbA1C, reduced cardiovascular risk, and improved obesity-related quality of life (43). In a study with longer follow-up, a longitudinal cohort of 1,048 patients undergoing LRYGB demonstrated a significantly lower CV risk from the first year after surgery that was sustained to the fifth postoperative year. After 5 years, the amelioration of the lipid profile itself yielded to a $27 \%$ reduction of $\mathrm{CV}$ risk $(\mathrm{P}<0.001)(44)$.

In another randomized control trial, patients with class II or III obesity and T2D for at least 5 years were randomly assigned to LRYGB, BPD or medical treatment. The primary endpoint was the rate of diabetes remission at 2 years, but evaluating cardiovascular risks was one of the secondary endpoints. Both surgical procedures were associated with significantly lower plasma lipids, cardiovascular risk, and medication use. Five major complications of diabetes (including one fatal myocardial infarction) arose in four (27\%) patients in the medical group compared with only one complication in the gastric bypass group and no complications in the BPD group. Although the BPD group appeared to have lower CVD risk compared to LRYGB, the statistical analysis was focused on comparison of the surgical $v s$. nonsurgical groups, and there was no statistical analysis to determine if there was a significant difference between the two surgical procedures (41).

The single anastomosis duodeno-ileal bypass with laparoscopic vertical sleeve gastrectomy (SADI-S) has been shown to have even better CVD risk reduction compared to LRYGB. Torres et al. reviewed data from 149 patients undergoing LRYGB compared to 106 patients undergoing SADI-S. Mid-term follow-up of 3 years demonstrates better weight loss and improvement of blood pressure, lipid profile, and insulin resistance with SADI-S than with LRYGB. Long-term follow up is needed to determine if this difference remains significant (45).

Prachand et al. reported that DS provides superior resolution of T2D, HTN, dyslipidemia compared to LRYGB. Prospectively collected data in 350 patients (198 and 152 patients underwent DS and LRYGB, respectively) revealed that diabetes, HTN, and dyslipidemia resolution was significantly greater at 3 years for DS compared to LRYGB (diabetes, $100 \%$ vs. 60\%; HTN, $68.0 \%$ vs. $38.6 \%$; dyslipidemia, $72 \%$ vs. 26.3 (46).

Alexandrides et al. also reported that patients undergoing a variant of BPD, BPD-LRYGB, experienced superior improvement of T2D, TC and TG and blood pressure compared to LRYGB patients. T2D resolved in $89 \%$ and 99\% of the cases following LRYGBP and BPD-LRYGBP, respectively. Both procedures lead to normalization of blood glucose, lipids, uric acid, liver enzymes and arterial pressure in the majority of patients, but this variant of BPD was more effective than LRYGB. Two years after BPDLRYGBP, $100 \%$ patients had blood glucose $<110 \mathrm{mg} / \mathrm{dL}$, 95\% had normal cholesterol, $92 \%$ normal triglycerides and $82 \%$ normal blood pressure. Patients undergoing LRYGBP with normal values were $66 \%, 33 \%, 78 \%$ and $44 \%$, respectively (47).

\section{Other cardiovascular benefits after bariatric surgery}

Atherosclerosis has been linked to circulating inflammation, and reduction in this circulating inflammation may explain 
Table 2 Relative CVD risk reduction determined in studies using risk prediction models

\begin{tabular}{|c|c|c|c|c|}
\hline Author & Year & Risk prediction model used & Procedure breakdown [n] & 10-year CVD relative risk reduction \\
\hline Raygor et al. (56) & 2019 & ACC/AHA ASCVD & LRYGB [438]; LVSG [98] & $54 \% ; 30 \%$ \\
\hline Wei et al. (57) & 2018 & UKPDS Risk Score & LRYGB [305]; LVSG [87]; SAGB [204] & $33 \% ; 50.5 \% ; 52.4 \%$ \\
\hline Gutierrez-Blanco et al. (62) & 2018 & FRS & LRYGB [779]; LVSG [220] & $36.3 \% ; 38.1 \%$ \\
\hline Piché et al. (63) & 2014 & FRS & BPD-DS [22 female]; BPD-DS [51 male] & $43 \% ; 33 \%$ \\
\hline Largent et al. (64) & 2013 & FRS & LAGB [697] & $29.6 \%$ \\
\hline Benaiges et al. (55) & 2011 & FRS; REGICOR & LRYGB [95]; LVSG [45] & $52.1 \%, 39.2 \% ; 51.2 \%, 40.6 \%$ \\
\hline Arterburn et al. (60) & 2009 & FRS & LRYGB [92] & $19.4 \%$ \\
\hline
\end{tabular}

FRS, Framingham Risk Score; ACC/AHA/ASCVD, American Colle of Cardiology/American Heart Association/Atherosclerotic Cardiovascular Disease; REGICOR, Registre Gironí del Cor; UKPDS, United Kingdom Prospective Diabetes Study; PROCAM, Prospective Cardiovascular Münster Model; LRYGB, Roux-en-Y gastric bypass; LVSG, sleeve gastrectomy; SAGB, single anastomosis gastric bypass; LAGB, laparoscopic adjustable gastric banding; BPD-DS, biliopancreatic diversion-duodenal switch.

improvements in the clinical indicators of CVD. Coronary calcification was shown to be significantly improved with sustained weight loss six years after LRYGB (48). Patients with obesity tend to have significantly increased carotid intima-media thickness (IMT) and impaired flow-mediated dilation (FMD). After surgery, a significant reduction of IMT and a significant improvement in FMD. Percent changes in BMI were associated with changes in IMT and FMD (49).

Metabolic and bariatric surgery has also been shown to decrease left ventricle (LV) mass and relative wall thickness, improve LV diastolic function, and decrease left atrium diameter. These changes were seen within six months after surgery. Measurement of right ventricle (RV) size and function, RV end-diastolic area and estimated RV systolic pressure are noted to improve significantly after LRYGB (50-53).

\section{Bariatric surgery improves CVD risk scores}

Several studies have more conclusively proven that bariatric surgery reduces CVD risk by demonstrating improved scores in accepted CVD risk models (54-64) (Table 2). Blanco $e t a l$. performed a retrospective review of the records of metabolic and bariatric surgery patients who met the criteria for calculating the 10-year ACC/AHA ASCVD risk score 10-year and the FRS. They demonstrated that both LVSG and LRYGB are equally effective in significantly reducing cardiovascular risk, with no statistical differences between the two procedures. Propensity score matching was used to match LVSG and LRYGB on demographic characteristics and co-morbidities. LVSG was performed in 159 patients compared with 60 patients undergoing LRYGB. At 12-month follow-up, the ASCVD 10-year score showed an absolute risk reduction of $3.9 \% \pm 6.5 \%$ in LVSG patients and $2.9 \% \pm 5.8 \%$ in LRYGB patients $(\mathrm{P}=0.3)$, resulting in a relative CVD risk reduction of $39 \%$ and $27.6 \%$, respectively. The FRS calculated an absolute risk reduction of $10.7 \%$ in LVSG patients and a $9.1 \%$ in LRYGB patients, resulting in a relative CVD risk reduction of $42.1 \%$ and $32.8 \%$, respectively. Significant decreases in the estimated heart age of $12.1 \pm 15.6$ years was seen in LVSG versus $9.2 \pm 9.6$ years in LRYGB $(\mathrm{P}=0.1)(54)$.

In a prospective cohort study of 140 consecutive patients (95 LRYGB and 45 LVSG) compared the 2 surgical intervention groups to study the percentage of excess weight loss, resolution and improvement/resolution of co-morbidities, and effect on CVD risk using both the FRS and the Registre Gironí del Cor (REGICOR) risk models. At one year, the overall $\mathrm{CV}$ risk decreased from $6.6 \%$ to $3.4 \%$ using the FRS and from $3.7 \%$ to $1.9 \%$ using the REGICOR score. No statistical differences were 
noted between the two surgical intervention groups. At 12 months, FRS decreased $3.4 \% \pm 2.2 \%$ for LRYGB versus $3.3 \% \pm 2.1 \%$ for LVSG $(\mathrm{P}=0.872)$, and the REGICOR score decreased $1.9 \% \pm 1.5 \%$ versus $1.8 \% \pm 1.6 \%$, respectively $(\mathrm{P}=0.813)$. The improvement, or resolution, of hypercholesterolemia was higher in the LRYGB group compared to the LVSG group (55).

Using the ACC/AHA ASCVD Pooled Cohort Risk Calculator, Raygor et al. looked at 10-year and lifetime ASCVD risks before and 1 year after metabolic and bariatric surgery for patients aged 40-78 who underwent LRYGB or LVSG. They demonstrated that patients who undergo LRYGB may experience greater cardiovascular risk reduction relative to counterparts who undergo LVSG. Of the 536 patients who underwent metabolic and bariatric surgery, 438 underwent LRYGB and 98 underwent LVSG. Significantly lower 10-year ASCVD risk for LRYGB and LVSG was $1.7 \% \pm 3.5 \%$ vs. $0.8 \% \pm 2.4 \%(\mathrm{P}<0.001)$, and lifetime ASCVD risk was $11 \% \pm 23 \%$ vs. $0 \% \pm 12 \%$ $(\mathrm{P}<0.001)$, compared to baseline measurements. Relative risk reduction was $54 \%$ in LRYGB patients and $30 \%$ in LVSG patients. The authors concluded that patients who underwent LRYGB experienced greater reductions in 10-year and lifetime ASCVD risks from baseline to 1 year after surgery than patients who underwent LVSG. Greater risk reduction was seen in patients who were females, age $<60$ years, and with a BMI of $35-50 \mathrm{~kg} / \mathrm{m}^{2}$ (56).

In a single institution review from China, Wei et al. looked at cerebral and coronary heart disease risk calculated using The UK Prospective Diabetes Study risk engine. The CVD risk reduction seen was up to 50\% and LRYGB appeared to have a greater effect on CVD risk reduction than LVSG one year after surgery. Outcomes were assessed in 392 patients (60\% female) who had undergone LVSG $(n=87)$ or LRYGB $(n=305)$ for treatment of T2D with one-year follow-up. One year following surgery, the 10 year CHD risk were significantly reduced from $8.8 \%$ to $5.9 \%$ in LVSG patients, and from $10.1 \%$ to $5 \%$ in LRYGB patients, representing a $33 \%$ and $50.5 \%$ relative CVD risk reduction, respectively. Ten-year risks for fatal CHD events significantly decreased as well $(38.1 \%$ vs. $55.4 \%$, respectively). Similar CVD risk reduction was seen in patients with $\mathrm{BMI} \geq 35$ and $\mathrm{BMI}<35$ one year after surgery. CVD risk was also calculated in 204 patients undergoing single anastomosis gastric bypass, demonstrating a 10 -year CHD risk reduction of $52.4 \%$ and a 10 -year fatal CHD event risk reduction of $59.5 \%$ (57).

Torquati et al. performed a single institution analysis of the change in CVD risk factors using the FRS to calculate the predicted 10-year absolute and relative risk of CHD in 500 patients undergoing LRYGB. The risk predicted by the FRS was then compared with the actual incidence of CHD events of the cohort. Compared with baseline, the average 10 -year absolute risk of cardiac events decreased from $5.4 \%$ at baseline to $2.7 \%$ at 1 year after operation. A similar risk reduction was observed in subgroups defined by diabetes status and gender. LRYGB decreased absolute risk of cardiac events by a mean of $63 \%$ in patients with diabetes, and $56 \%$ in male patients. At 5 years, the actual incidence of $\mathrm{CHD}$ events was considerably lower than the predicted rate prior to LRYGB (58).

Batsis et al. performed a systematic review and calculated 10-year CVD risk using FRS and Prospective Cardiovascular Munster Heart Study (PROCAM) risk score. The analysis comprised a validation cohort of 197 patients who underwent LRYGB and 163 control patients. The surgery group realized a significantly reduced FRS ( $7.0 \%$ to $3.5 \%)$, a $50 \%$ risk reduction, compared with $8.5 \%$ risk reduction seen with nonsurgical control patients. PROCAM risk in the bariatric surgery group decreased from $4.1 \%$ to $2.0 \%(\mathrm{P}<0.001)$, whereas the control group exhibited only a modest $13.6 \%$ risk reduction (59).

Arterburn et al. estimated cardiovascular risk using the FRS in 92 patients undergoing LRYGB. The predicted baseline 10 -year CVD risk was $6.7 \%$. At 6 and 12 months, the predicted risk had decreased to $5.2 \%$ and $5.4 \%$, respectively with an absolute risk reduction of $1.3 \%$, suggesting that 77 patients with class II or III obesity would have to undergo LRYGB to avert one new case of CVD over the ensuing 10 years (60).

Benotti et al. used the FRS to demonstrate LRYGB is associated with a reduced risk of myocardial infarction, stroke and the development of congestive heart failure (CHF). In a matched cohort study comparing 1,724 LRYGB patients and 1,724 nonsurgical patients followed for up to 12 years after surgery (median, 6.3 years), a statistically significant reduction in major composite cardiovascular events was seen in the LRYGB group, noting a $42 \%$ risk reduction of severe composite cardiovascular events. After LRYGB, significant improvement was seen in the 10 -year CVD risk score, TC, HDL, SBP, and T2D. The control group only realized an approximately $14 \%$ reduction (61).

Gutierrez-Blanco et al. demonstrated that LVSG has a positive impact in the reduction of the 10 -year CVD risk using the FRS in a retrospective review of all patients who underwent metabolic and bariatric surgery. The initial 
Framingham 10-year score risk was significantly higher in males compared with females, and after 12-month followup, a significant absolute risk reduction was seen in males $(11.58 \% ; \mathrm{P}<0.001)$ and $6.17 \%$ in females $(\mathrm{P}<0.001)$. The preoperative heart age was high in females and males $[69.23 \pm 15.72$ years and $73.55 \pm 13.55$ years, respectively $(\mathrm{P}=0.012)]$, and after 12 months it reduced 7.19 years in females $(\mathrm{P}<0.001)$ and 7.04 years in males $(\mathrm{P}<0.001)(62)$.

The 10-year predicted risk for CHD was estimated using the FRS in 73 patients who underwent biliopancreatic diversion with duodenal switch (BPD-DS) and compared with 33 nonsurgical control patients. Predicted risks were stratified into 3 groups: (I) high short-term predicted risk ( $\geq 10 \% 10$-year risk or diagnosed diabetes), (II) low shortterm ( $<10 \% 10$-year risk)/low lifetime predicted risk or (III) low short-term/high lifetime predicted risk. A significant reduction in HbA1C, Homeostatic Model Assessment of Insulin Resistance (HOMA-IR), all lipoprotein levels, and blood pressure was seen in the surgery group. The 10-year CHD predicted risk decreased by $43 \%$ in women and $33 \%$ in men, whereas the estimated CHD risk in the nonsurgical group did not change. Before surgery, none of the women and only $18 \%$ of men showed low short-term/low lifetime predicted risk, whereas a significant proportion of subjects had high short-term predicted risk $36 \%$ in women and $12 \%$ in men). Following surgery, $52 \%$ of women and $55 \%$ of men have a low short-term/low lifetime predicted risk (63).

Although LAGB accounts for approximately $7 \%$ of all bariatric procedures worldwide, and only $1 \%$ in the United States, studies have demonstrated significant long-term CVD risk reduction. Stable weight loss and significant improvement of cardiovascular risk profile were observed in patients with class I, II and III obesity after LAGB. The FRS was used to calculate estimated 10- and 30-year CVD risk reduction after LAGB in adult patients with obesity (BMI $\left.\geq 30 \mathrm{~kg} / \mathrm{m}^{2}\right)$. Surgery patients were propensity matched with nonsurgical patients. Ten- and 30-year estimated CVD risk significantly decreased from $10.8 \%$ to $7.6 \%$ and $44.34 \%$ to $32.30 \%(\mathrm{P}<0.0001)$, respectively, 12 to 15 months post-LAGB. In a cohort with lipid data $(\mathrm{n}=74)$, improvements in TC and HDL-C were also seen one-year post-LAGB (64).

\section{Bariatric surgery reduces cardiovascular events}

Several matched cohort studies reported the relationship between metabolic and bariatric surgery with major adverse cardiovascular events (MACE) in patients with T2D. Aminian et al. investigated adult patients with diabetes and obesity, matching one metabolic and bariatric surgery patient to five nonsurgical patients. $63 \%$ of the cohort underwent LRYGB and 32\% underwent LVSG. Patients in the surgical group experienced a 39\% reduction in first occurrence of all-cause mortality, coronary artery events, cerebrovascular events, heart failure, nephropathy, and atrial fibrillation. Additionally, when talking into account myocardial infraction, ischemic stroke and mortality as endpoints, patients in the surgical group realized a $41 \%$ risk reduction (65).

Fisher et al. looked at the incidence of coronary artery disease, cerebrovascular disease, and mortality in obese patients with type 2 diabetes undergoing metabolic and bariatric surgery compared to nonsurgical patients. The distribution of surgical patients included 76\% LRYGB, 17\% LVSG, and 7\% LAGB. At 5 years, surgery was associated with a $40 \%$ risk reduction of macrovascular events and a $67 \%$ risk reduction in all-cause mortality (66). The reported 2 to 3 times lower annual event rates reported by Fisher, compared to Aminian, may due to a lower-risk cohort.

The Swedish Obese Subjects (SOS) study is one of the largest prospective, nonrandomized controlled studies looking at outcomes between matched cohorts receiving metabolic and bariatric surgery and nonsurgical care. The surgery patients had significantly fewer CVD deaths: 20 events among 2,010 patients in the surgery group (1.0\%) versus 49 events among 2,037 patients in the control group $(2.4 \%)$, resulting in a $53 \%$ risk reduction. Additionally, the surgery group had significantly fewer first time CVD events (myocardial infarction or stroke, whichever came first) with $33 \%$ risk reduction. The same group also reported obese patients with T2D that included 343 surgical and 260 control patients. Metabolic and bariatric surgery was associated with significantly fewer macrovascular events than patients receiving nonsurgical care. The distribution of cases, however, were $66 \%$ vertical banded gastroplasty, with LAGB and LRYGB representing $18 \%$ and $13.2 \%$ of the surgical cohort, respectively $(67,68)$. Despite that, the study emphasizes the superior value of surgical, compared to nonoperative, management of obesity and its comorbidities.

In a study specific to patients receiving a LAGB, the 10-year probability of myocardial infarction was calculated using the PROCAM risk score and then compared to observed occurrences over time. A total of 318 patients (58 men and 260 women) underwent LAGB and follow-up was $12.7 \pm 1.5$ years. 
A significant reduction was observed in blood glucose, TC, triglycerides, and SBP and DBP pressure at shortterm evaluations and confirmed after long term evaluations. HDL-C was unchanged at 12-18 months, but significantly increased at 12.7 years. Five coronary events $(1.6 \%)$ were recorded during long-term follow-up, which was slightly lower than the expected rate $(2.0 \% \pm 4.9 \%)(69)$.

\section{Conclusions}

A substantial, and sustained, decrease in body weight after metabolic and bariatric surgery is associated with a significant reduction of cardiovascular risk factors such as T2D, HTN and hypertriglyceridemia. Several studies have demonstrated equally effective outcomes regardless of which procedure is performed, while others suggest that patients undergoing LRYGB, and even DS, experience a greater reduction in CVD risk. The comparison studies to date are insufficient to definitively conclude which procedure provides the best CVD risk reduction in the short, or long, term. However, DS appears to have better long-term reduction in CVD risk factors, followed by LRYGB, LVSG and LAGB. Randomized control trials are required to better define and confirm which procedure is indeed the most superior at reducing CVD risk.

\section{Acknowledgments}

Funding: None.

\section{Footnote}

Provenance and Peer Review: This article was commissioned by the Guest Editor (Muhammed Ashraf Memon) for the focused issue "Bariatric Surgery" published in Annals of Translational Medicine. The article was sent for external peer review organized by the Guest Editor and the editorial office.

Conflicts of Interest: The focused issue "Bariatric Surgery" was commissioned by the editorial office without any funding or sponsorship. The authors have no conflicts of interest to declare.

Ethical Statement: The authors are accountable for all aspects of the work in ensuring that questions related to the accuracy or integrity of any part of the work are appropriately investigated and resolved.
Open Access Statement: This is an Open Access article distributed in accordance with the Creative Commons Attribution-NonCommercial-NoDerivs 4.0 International License (CC BY-NC-ND 4.0), which permits the noncommercial replication and distribution of the article with the strict proviso that no changes or edits are made and the original work is properly cited (including links to both the formal publication through the relevant DOI and the license). See: https://creativecommons.org/licenses/by-nc-nd/4.0/.

\section{References}

1. Available online: https://www.who.int/en/news-room/ fact-sheets/detail/cardiovascular-diseases-(cvds). Website accessed 1 Sept 2019.

2. Goldstein DJ. Beneficial Health Effects of Modest Weight Loss. Int J Obes Relat Metab Disord 1992;16:397-415.

3. Look AHEAD Research Group. Eight-year weight losses with an intensive lifestyle intervention: the look AHEAD study. Obesity (Silver Spring) 2014;22:5-13.

4. Li G, Zhang P, Wang J, et al. The long-term effect of lifestyle interventions to prevent diabetes in the China Da Qing Diabetes Prevention Study: a 20-year follow-up study. Lancet 2008;371:1783-9.

5. Uusitupa M, Peltonen M, Lindström J, et al. Ten-year mortality and cardiovascular morbidity in the Finnish Diabetes Prevention Study--secondary analysis of the randomized trial. PLoS One 2009;4:e5656.

6. Eliasson B, Liakopoulos V, Franzén S, et al. Cardiovascular disease and mortality in patients with type 2 diabetes after bariatric surgery in Sweden: a nationwide, matched, observational cohort study. Lancet Diabetes Endocrinol 2015;3:847-54.

7. Lloyd-Jones DM. Cardiovascular risk prediction: basic concepts, current status, and future directions. Circulation 2010;121:1768-77.

8. Conroy RM, Pyörälä K, Fitzgerald AP, et al. Estimation of ten-year risk of fatal cardiovascular disease in Europe: the SCORE project. Eur Heart J 2003;24:987-1003.

9. Hippisley-Cox J, Coupland C, Vinogradova Y, et al. Derivation and validation of QRISK, a new cardiovascular disease risk score for the United Kingdom: prospective open cohort study. BMJ 2007;335:136.

10. Ridker PM, Buring JE, Rifai N, et al. Development and validation of improved algorithms for the assessment of global cardiovascular risk in women: the Reynolds Risk Score. JAMA 2007;297:611-9.

11. Ridker PM, Paynter NP, Rifai N, et al. C-reactive protein 
and parental history improve global cardiovascular risk prediction: the Reynolds Risk Score for men. Circulation 2008;118:2243-51.

12. Stevens RJ, Kothari V, Adler AI, et al. The UKPDS risk engine: a model for the risk of coronary heart disease in Type II diabetes (UKPDS 56). Clin Sci (Lond) 2001;101:671-9.

13. Marrugat J, D'Agostino R, Sullivan L, et al. An adaptation of the Framingham coronary heart disease risk function to European Mediterranean areas. J Epidemiol Community Health 2003;57:634-8.

14. National Cholesterol Education Program (NCEP) Expert Panel on Detection, Evaluation, and Treatment of High Blood Cholesterol in Adults (Adult Treatment Panel III). Third Report of the National Cholesterol Education Program (NCEP) Expert Panel on Detection, Evaluation, and Treatment of High Blood Cholesterol in Adults (Adult Treatment Panel III) final report. Circulation 2002;106:3143-421.

15. Kannel WB, Feinleib M, McNamara PM, et al. An investigation of coronary heart disease in families. The Framingham offspring study. Am J Epidemiol 1979;110:281-90.

16. D'Agostino RB Sr, Vasan RS, Pencina MJ, et al. General cardiovascular risk profile for use in primary care: the Framingham Heart Study. Circulation 2008;117:743-53.

17. Goff DC, Lloyd-Jones DM, Bennett G, et al 2013 ACC/ AHA guideline on the assessment of cardiovascular risk: a report of the American College of Cardiology/American Heart Task Force on Practice Guidelines Circulation 2014;129:S49-73.

18. The Atherosclerosis Risk in Communities (ARIC) Study: design and objectives. The ARIC investigators. Am J Epidemiol 1989;129:687-702.

19. Friedman GD, Cutter GR, Donahue RP, et al. CARDIA: study design, recruitment, and some characteristics of the examined subjects. J Clin Epidemiol 1988;41:1105-16.

20. Fried LP, Borhani NO, Enright P, et al. The Cardiovascular Health Study: design and rationale. Ann Epidemiol 1991;1:263-76.

21. Pasternak RC, Abrams J, Greenland P, et al. 34th Bethesda Conference: Task force \#1--Identification of coronary heart disease risk: is there a detection gap? J Am Coll Cardiol 2003;41:1863-74.

22. Bays H, Kothari SN, Azagury DE, et al. Lipids and bariatric procedures Part 2 of 2: scientific statement from the American Society for Metabolic and Bariatric Surgery (ASMBS), the National Lipid Association(NLA), and
Obesity Medicine Association (OMA). Surg Obes Relat Dis 2016;12:468-95.

23. Piercy KL, Troiano RP. Physical Activity Guidelines for Americans From the US Department of Health and Human Services. Circ Cardiovasc Qual Outcomes 2018;11:e005263.

24. Warburton DE, Nicol CW, Bredin SS. Health benefits of physical activity: the evidence. CMAJ 2006;174:801-9.

25. Nedeljkovic-Arsenovic O, Banovic M, Radenkovic D, et al. The Amount of Weight Loss Six Months after Bariatric Surgery: It Makes a Difference. Obes Facts 2019;12:281-90.

26. Herring LY, Stevinson C, Davies MJ, et al. Changes in physical activity behaviour and physical function after bariatric surgery: a systematic review and meta-analysis. Obes Rev 2016;17:250-61.

27. Kissebah AH, Vydelingum N, Murray R, et al. Relation of body fat distribution to metabolic complications of obesity. J Clin Endocrinol Metab 1982;54:254-60.

28. Krotkiewski M, Björntorp P, Sjöström L, et al. Impact of obesity on metabolism in men and women. Importance of regional adipose tissue distribution. J Clin Invest 1983;72:1150-62.

29. Neeland IJ, Ross R, Després JP, et al. Visceral and ectopic fat, atherosclerosis, and cardiometabolic disease: a position statement. Lancet Diabetes Endocrinol 2019;7:715-25.

30. Després JP. Body fat distribution and risk of cardiovascular disease: an update. Circulation 2012;126:1301-13.

31. Batra A, Siegmund B. The role of visceral fat. Dig Dis 2012;30:70-4.

32. Askarpour M, Khani D, Sheikhi A, et al. Effect of Bariatric Surgery on Serum Inflammatory Factors of Obese Patients: a Systematic Review and Meta-Analysis. Obes Surg 2019;29:2631-47.

33. Stolberg CR, Mundbjerg LH, Funch-Jensen P, et al. Effects of gastric bypass surgery followed by supervised physical training on inflammation and endothelial function: A randomized controlled trial. Atherosclerosis 2018;273:37-44.

34. Gebhart A, Young M, Villamere J, et al. Changes in highsensitivity C-reactive protein levels after laparoscopic gastric stapling procedures versus laparoscopic gastric banding. Am Surg 2014;80:1044-8.

35. Yayan J. Erythrocyte sedimentation rate as a marker for coronary heart disease. Vasc Health Risk Manag 2012;8:219-23.

36. Bacci V, Basso MS, Greco F, et al. Modifications of metabolic and cardiovascular risk factors after weight 


\section{Page 10 of 11}

loss induced by laparoscopic gastric banding. Obes Surg 2002;12:77-82.

37. Angrisani L, Santonicola A, Iovino P, et al. Bariatric Surgery and Endoluminal Procedures: IFSO Worldwide Survey 2014. Obes Surg 2017;27:2279-89.

38. English WJ, DeMaria EJ, Brethauer SA, et al. American Society for Metabolic and Bariatric Surgery estimation of metabolic and bariatric procedures performed in the United States in 2016. Surg Obes Relat Dis 2018;14:259-63.

39. Heneghan HM, Meron-Eldar S, Brethauer SA, et al. Effect of bariatric surgery on cardiovascular risk profile. Am J Cardiol 2011;108:1499-507.

40. Schauer PR, Bhatt DL, Kirwan JP, et al. Bariatric Surgery versus Intensive Medical Therapy for Diabetes - 5-Year Outcomes. N Engl J Med 2017;376:641-51.

41. Mingrone G, Panunzi S, De Gaetano A, et al. Bariatricmetabolic surgery versus conventional medical treatment in obese patients with type 2 diabetes: 5 year follow-up of an open-label, single-centre, randomised controlled trial. Lancet 2015;386:964-73.

42. Adams TD, Davidson LE, Litwin SE, et al. Weight and Metabolic Outcomes 12 Years after Gastric Bypass. N Engl J Med 2017;377:1143-55.

43. Simonson DC, Halperin F, Foster K, et al. Clinical and Patient-Centered Outcomes in Obese Patients With Type 2 Diabetes 3 Years After Randomization to Rouxen-Y Gastric Bypass Surgery Versus Intensive Lifestyle Management: The SLIMM-T2D Study. Diabetes Care 2018;41:670-9.

44. Gero D, Favre L, Allemann P, et al. Laparoscopic RouxEn-Y Gastric Bypass Improves Lipid Profile and Decreases Cardiovascular Risk: a 5-Year Longitudinal Cohort Study of 1048 Patients. Obes Surg 2018;28:805-11.

45. Torres A, Rubio MA, Ramos-Leví AM, et al. Cardiovascular Risk Factors After Single Anastomosis Duodeno-Ileal Bypass with Sleeve Gastrectomy (SADI-S): A New Effective Therapeutic Approach? Curr Atheroscler Rep 2017;19:58.

46. Prachand VN, Ward M, Alverdy JC. Duodenal switch provides superior resolution of metabolic comorbidities independent of weight loss in the super-obese (BMI > or $=50 \mathrm{~kg} / \mathrm{m} 2$ ) compared with gastric bypass. J Gastrointest Surg 2010;14:211-20.

47. Alexandrides TK, Skroubis G, Kalfarentzos F. Resolution of diabetes mellitus and metabolic syndrome following Roux-en-Y gastric bypass and a variant of biliopancreatic diversion in patients with morbid obesity. Obes Surg 2007;17:176-84.
English et al. Cardiovascular risk reduction after bariatric surgery

48. Priester T, Ault TG, Davidson L, et al. Coronary calcium scores 6 years after bariatric surgery. Obes Surg 2015;25:90-6.

49. Lupoli R, Di Minno MN, Guidone C, et al. Effects of bariatric surgery on markers of subclinical atherosclerosis and endothelial function: a meta-analysis of literature studies. Int J Obes (Lond) 2016;40:395-402

50. Cuspidi C, Rescaldani M, Tadic M, et al. Effects of bariatric surgery on cardiac structure and function: a systematic review and meta-analysis. Am J Hypertens 2014;27:146-56.

51. Kurnicka K, Domienik-Karłowicz J, Lichodziejewska B, et al. Improvement of left ventricular diastolic function and left heart morphology in young women with morbid obesity six months after bariatric surgery. Cardiol J 2018;25:97-105.

52. Pugh ME, Newman JH, Williams DB, et al. Hemodynamic improvement of pulmonary arterial hypertension after bariatric surgery: potential role for metabolic regulation. Diabetes Care 2013;36:e32-3.

53. Garza CA, Pellikka PA, Somers VK, et al. Structural and functional changes in left and right ventricles after major weight loss following bariatric surgery for morbid obesity. Am J Cardiol 2010;105:550-6.

54. Blanco DG, Funes DR, Giambartolomei G, et al. Laparoscopic sleeve gastrectomy versus Roux-en-Y gastric bypass in cardiovascular risk reduction: A match control study. Surg Obes Relat Dis 2019;15:14-20

55. Benaiges D, Goday A, Ramon JM, et al. Laparoscopic sleeve gastrectomy and laparoscopic gastric bypass are equally effective for reduction of cardiovascular risk in severely obese patients at one year of follow-up. Surg Obes Relat Dis 2011;7:575-80.

56. Raygor V, Garcia L, Maron DJ, et al. The Comparative Effect of Roux-en-Y Gastric Bypass and Sleeve Gastrectomy on 10-Year and Lifetime Atherosclerotic Cardiovascular Disease Risk. Obes Surg 2019;29:3111-7.

57. Wei JH, Chou RH, Huang PH, et al. Metabolic surgery ameliorates cardiovascular risk in obese diabetic patients: Influence of different surgical procedures. Surg Obes Relat Dis 2018;14:1832-40.

58. Torquati A, Wright K, Melvin W, et al. Effect of gastric bypass operation on Framingham and actual risk of cardiovascular events in class II to III obesity. J Am Coll Surg 2007;204:776-82.

59. Batsis JA, Sarr MG, Collazo-Clavell ML, et al. Cardiovascular risk after bariatric surgery for obesity. Am J Cardiol 2008;102:930-7. 
60. Arterburn D, Schauer DP, Wise RE, et al. Change in predicted 10-year cardiovascular risk following laparoscopic Roux-en-Y gastric bypass surgery. Obes Surg 2009; 19:184-9.

61. Benotti PN, Wood GC, Carey DJ, et al. Gastric Bypass Surgery Produces a Durable Reduction in Cardiovascular Disease Risk Factors and Reduces the Long-Term Risks of Congestive Heart Failure. J Am Heart Assoc 2017. doi: 10.1161/JAHA.116.005126.

62. Gutierrez-Blanco D, Funes-Romero D, Madiraju S, et al. Reduction of Framingham BMI score after rapid weight loss in severely obese subjects undergoing sleeve gastrectomy: a single institution experience. Surg Endosc 2018;32:1248-54.

63. Piché MÈ, Martin J, Cianflone K, et al. Changes in predicted cardiovascular disease risk after biliopancreatic diversion surgery in severely obese patients. Metabolism 2014;63:79-86.

64. Largent JA, Vasey J, Bessonova L, et al. Reduction in Framingham risk of cardiovascular disease in obese patients undergoing laparoscopic adjustable gastric

Cite this article as: English WJ, Spann MD, Aher CV, Williams DB. Cardiovascular risk reduction following metabolic and bariatric surgery. Ann Transl Med 2020;8(Suppl 1):S12. doi: 10.21037/atm.2020.01.88 banding. Adv Ther 2013;30:684-96.

65. Aminian A, Zajichek A, Arterburn DE, et al. Association of Metabolic Surgery with Major Adverse Cardiovascular Outcomes in Patients with Type 2 Diabetes and Obesity. JAMA 2019. [Epub ahead of print].

66. Fisher DP, Johnson E, Haneuse S, et al. Association Between Bariatric Surgery and Macrovascular Disease Outcomes in Patients with Type 2 Diabetes and Severe Obesity. JAMA 2018;320:1570-82.

67. Sjöström L, Peltonen M, Jacobson P, et al. Bariatric surgery and long-term cardiovascular events. JAMA 2012;307:56-65.

68. Sjöström L, Peltonen M, Jacobson P, et al. Association of bariatric surgery with long-term remission of type 2 diabetes and with microvascular and macrovascular complications. JAMA 2014;311:2297-304.

69. Busetto L, De Stefano F, Pigozzo S, et al. Long-term cardiovascular risk and coronary events in morbidly obese patients treated with laparoscopic gastric banding. Surg Obes Relat Dis 2014;10:112-20. 